\title{
Pseudorange error analysis for precise indoor positioning system
}

\author{
Marek Pola, Pavel Bezoušek *
}

\begin{abstract}
There is a currently developed system of a transmitter indoor localization intended for fire fighters or members of rescue corps. In this system the transmitter of an ultra-wideband orthogonal frequency-division multiplexing signal position is determined by the time difference of arrival method. The position measurement accuracy highly depends on the directpath signal time of arrival estimation accuracy which is degraded by severe multipath in complicated environments such as buildings. The aim of this article is to assess errors in the direct-path signal time of arrival determination caused by multipath signal propagation and noise. Two methods of the direct-path signal time of arrival estimation are compared here: the cross correlation method and the spectral estimation method.
\end{abstract}

K e y w o r ds: ultra-wideband (UWB), time difference of arrival (TDOA), orthogonal frequency-division multiplexing (OFDM), Cramer-Rao bound (CRB), cross-correlation function (CCF)

\section{Introduction}

The aim of this article is to analyze errors of pseudoranges measured between the transmitter and receiver for the system to locate the position of people in a complicated environment [18]. The analyzed system is designed to locate rescue team members or robotic exploration platforms in closed spaces at critical and life-threatening conditions and situations occurring during the fires of houses and industrial buildings or in natural disasters. Therefore our goal is to determine positions of cooperating objects equipped with a technical devices permitting their identification.

Currently, there are a large number of research papers dealing with indoor transmitter localization mostly utilizing ultra-wideband (UWB) radio frequency signal - summarized here [2]. The system we have developed, uses wideband OFDM signal for accurate measurement of the signals arrival times sent by a transmitter and detected by several receiver stations placed outside the monitored area [1]. The transmitter location is then determined by the TDOA method [1]. In this article we will pay a closer attention to the analysis of errors of arrival measurements of the directpath signal due to the multipath and noise. Here we compare two methods of the time of arrival estimation, namely: the method, based on the cross-correlation function of the received and the transmitted signal (the correlation estimator) and the modified covariance method of the spectrum of the received signal estimation (hereinafter referred to as the spectral estimator).

\section{Cramer-Rao bound}

The lower limit of estimation variance (error) of a nonrandom parameter $\lambda$ (in our case of the signal time of arrival) based on the measurement of some variable $X$ through any unbiased estimator (estimation methods) is the so-called Cramer-Rao bound CRB (CRLB) given by either form $([3,4])$

$$
\begin{array}{r}
\operatorname{CRB}(\lambda)=\frac{1}{E\left[\left\{\frac{\partial}{\partial \lambda} \ln p(X \mid \lambda)\right\}^{2}\right]_{X}} \\
\operatorname{CRB}(\lambda)=\frac{1}{E\left[\frac{\partial^{2}}{\partial \lambda^{2}} \ln p(X \mid \lambda)\right]_{X}}
\end{array}
$$

where $\lambda$ is estimated parameter, $X$ is measured value, $p(X \mid \lambda)$ is likelihood function of estimator $\hat{\lambda}(X)$ and $E[y]$ is the mean statistic value of $y$.

The lower limit of the mean square error $\sigma_{\tau 0}$ of delay $\tau_{0}$ measurement of direct signal is then equal to the square root of the CRB. The CRB limit estimate of the time of arrival of signals has been derived in the literature many times under different conditions. Frequently a large signal to noise ratio was assumed. In the case of a one-way signal propagation between the transmitter and receiver this limit can be expressed $e g$; by [5] as follows

$$
\begin{aligned}
& \operatorname{CRLB}(\hat{\tau})=\frac{1}{2 \operatorname{SNR} \beta^{2}}, \\
& \beta^{2}=\frac{1}{E_{p}} 4 \pi^{2} \int_{-\infty}^{\infty} f^{2}|S(f)|^{2} \mathrm{~d} f
\end{aligned}
$$

where SNR is signal to noise ratio in the place of measurement, $E_{p}$ is signal energy, $|S(f)|^{2}$ is energy spectral density of transmitted signal.

In our case, considering a rectangular spectrum of the complex envelope: in the band $f \in<-b / 2 ;+b / 2>$ we get

$$
\sigma_{\tau}=\sqrt{\operatorname{CRLB}(\hat{\tau})}=\frac{1}{\frac{\pi}{\sqrt{3}} b \sqrt{2 \mathrm{SNR}}}
$$

University of Pardubice, Department of Electrical Engineering, the Czech Republic, marek.pola@upce.cz, pavel.bezousek@upce.cz 
In the multipath situation the formula (4) could be used when replicas are sufficiently separated from the direct-path signal, $i e$ when the time separation $\delta \tau$ of the neighbor replica meets the following condition: $b \delta \tau>>$ 1 .

In the case of close replicas the CRB formula is very complicated. For a determined replica with complex amplitudes delays distribution the relationship (at a high SNR) was published by authors of [6]. They showed that a significant influence on the time of arrival estimation had mainly the closest replicas in the range of the method time resolution $1 / b$.

Although the Maximum Likelihood Estimator (MLE) meets the $\mathrm{CRB}$ as a rule, the application of this procedure is very complicated in practice, because it leads to a multidimensional nonlinear optimization. Therefore, typically suboptimal estimators are used.

\section{Correlation estimator}

This estimator determines the delay of the incoming signal by searching for the maximum of cross-correlation function $B_{1,2}(\theta)$ from the complex envelopes of transmitted and received signal

$$
\begin{aligned}
& B_{1,2}(\theta)=\int_{-\infty}^{\infty} V_{1}^{*}(t) V_{2}(t+\theta) \mathrm{d} t= \\
& =\int_{-\infty}^{\infty}\left[V_{1 \mid}^{*}(t) \sum_{l=1}^{L} a_{l} V_{1}\left(t-\tau_{l}+\theta\right)+V_{1}^{*}(t) \nu(t+\theta)\right] \mathrm{d} t= \\
& =\sum_{l=1}^{L} \int_{-\infty}^{+\infty} a_{l} V_{1}^{*}(t) V_{1}\left(t-\tau_{l}+\theta\right) \mathrm{d} t+B_{1, \nu}(\theta)= \\
& =\sum_{l=1}^{L} a_{l} B_{1}\left(\theta-\tau_{l}\right)+B_{1, \nu}(\theta)
\end{aligned}
$$

$$
\begin{gathered}
V_{1}(t)=\sqrt{\frac{2 S_{1}}{M+1}} \sum_{n=-\frac{M}{2}}^{\frac{M}{2}} \exp \left(j n \omega_{0} t\right), \\
B_{1}(\theta)=\int_{-\infty}^{\infty} V_{1}^{*}(t) V_{1}(t+\theta) \mathrm{d} t
\end{gathered}
$$

where $V_{1}(t), V_{2}(t), \nu(t)$ are complex envelopes of transmitted, received signal and noise, $B(\theta)$ is the correlation of $V_{1}(t), B_{1,2}(\theta)$ is cross the correlation of $V_{1}(t)$ and $V_{2}(t), B_{1, \nu}(\theta)$ is the cross correlation of $V_{1}(t)$ and $\nu(t)$.

Therefore, our estimator $\eta$ can be defined ( $\eta$ is $l$-th root)

$$
\frac{\partial B_{1,2}(\tau)}{\partial \tau}=0 \text { and } \frac{\partial^{2} B_{1,2}(\tau)}{\partial \tau^{2}}<0
$$

\subsection{Single replica propagation linear approach}

In the case of one replica and a high SNR according to (7) can be linearized

$$
\begin{array}{r}
\frac{\partial B_{1}\left(\theta-\tau_{1}\right)}{\partial \theta} \cong \frac{\partial^{2} B_{1}\left(\theta-\tau_{1}\right)}{\partial \theta^{2}} \Delta \tau_{1} \Rightarrow \\
\left.a_{1} \Delta \tau_{1} \frac{\partial^{2} B_{1}\left(\theta-\tau_{1}\right)}{\partial \theta^{2}}\right|_{\theta=\tau_{1}}+\left.\frac{B_{1, \nu}(\theta)}{\partial \theta}\right|_{\theta=\tau_{1}}=0
\end{array}
$$

hence

$$
\begin{gathered}
\Delta \tau_{1}=-\frac{\left.\frac{B_{1, \nu}(\theta)}{\partial \theta}\right|_{\theta=\tau_{1}}}{\left.a_{1} \frac{\partial^{2} B_{1}\left(\theta-\tau_{1}\right)}{\partial \theta^{2}}\right|_{\theta=\tau_{1}}} \Rightarrow \\
\Rightarrow D\left[\Delta \tau_{1}\right]=-\frac{D\left[\left.\frac{B_{1, \nu}(\theta)}{\partial \theta}\right|_{\theta=\tau_{1}}\right]}{\left[\left.a_{1} \frac{\partial^{2} B_{1}(\theta)}{\partial \theta^{2}}\right|_{\theta=0}\right]^{2}}
\end{gathered}
$$

Given that our estimator is a linear, variance of the time of arrival measurement achieves the limits of CRB for this signal. On the other hand, it should be noted that estimator could be linearized only for sufficiently large signal to noise ratio.

\subsection{Multi replica propagation linear approach}

Cross correlation functions $B_{12}(\theta)$ of the received signal with the transmitted signal is a linear transformation of the received signal and therefore, if the signal comprises from several received replicas of the transmitted signal and noise, this cross correlation function is equal to a linear combination of shifted autocorrelation functions of the transmitted signal and cross-correlation function of noise and transmitted signal $B_{1}(\theta)$. For example, in the case of only two replicas

$$
\begin{aligned}
& \frac{\partial B_{1,2}(\theta)}{\partial \theta}=a_{1} \frac{\partial B_{1}\left(\theta-\tau_{1}\right)}{\partial \theta}+ \\
& +a_{2} \frac{\partial B_{1}\left(\theta-\tau_{2}\right)}{\partial \theta}+\frac{B_{1, \nu}(\theta)}{\partial \theta}=0
\end{aligned}
$$

where $a_{1}, a_{2}$ are amplitudes of first (LOS) and second replica. For small amplitudes of second $a_{2}$ replica, (10) can be linearized

$$
\begin{aligned}
& \left.a_{1} \Delta \tau_{1} \frac{\partial^{2} B_{1}\left(\theta-\tau_{1}\right)}{\partial \theta^{2}}\right|_{\theta=\tau_{1}}+\left.a_{2} \frac{\partial B_{1}\left(\theta-\tau_{2}\right)}{\partial \theta}\right|_{\theta=\tau_{1} q} \\
& +\left.\frac{B_{1, \nu}(\theta)}{\partial \theta}\right|_{\theta=\tau_{1}}=0 \\
& \left.a_{1} \Delta \tau_{1} \frac{\partial^{2} B_{1}(\theta)}{\partial \theta^{2}}\right|_{\theta=0}+\left.a_{2} \frac{\partial B_{1}\left(\theta-\left(\tau_{2}-\tau_{1}\right)\right)}{\partial \theta}\right|_{\theta=0}+ \\
& +\left.\frac{B_{1, \nu}(\theta)}{\partial \theta}\right|_{\theta=\tau_{1}}=0
\end{aligned}
$$




$$
\Delta \tau_{1}=-\frac{\left.a_{2} \frac{\partial B_{1}\left[\theta-\left(\tau_{2}-\tau_{1}\right)\right]}{\partial \theta}\right|_{\theta=0}+\left.\frac{B_{1, \nu}(\theta)}{\partial \theta}\right|_{\theta=\tau_{1}}}{\left.a_{1} \frac{\partial^{2} B_{1}(\theta)}{\partial \theta^{2}}\right|_{\theta=0}}
$$

In case of defined value $\tau_{21}=\tau_{2}-\tau_{1}$

$$
\begin{aligned}
& E\left[\Delta \tau_{1}\right]=-E\left[\frac{a_{2}}{a_{1}}\right] \frac{\left.\frac{\partial B_{1}\left(\theta-\tau_{21}\right)}{\partial \theta}\right|_{\theta=0}}{\left.\frac{\partial^{2} B_{1}(\theta)}{\partial \theta^{2}}\right|_{\theta=0}}[\mathrm{~s}] \\
& \sigma_{\Delta \tau_{1}}=\sqrt{E\left[\left(\Delta \tau_{1}-E\left[\Delta \tau_{1}\right]\right)^{2}\right]}= \\
& =\sqrt{E\left[\left(\frac{a_{2}}{a_{1}}-E\left[\frac{a_{2}}{a_{1}}\right]\right)^{2}\right]\left|\frac{\left.\frac{\partial B_{1}\left(\theta-\tau_{21}\right)}{\partial \theta}\right|_{\theta=0}}{\left.\frac{\partial^{2} B_{1}(\theta)}{\partial \theta^{2}}\right|_{\theta=0}}\right|}
\end{aligned}
$$

In the case of small amplitude $a_{2}$, and zero mean ratios $a_{2} / a_{1}$ of complex amplitudes of replicas the estimator will be unbiased. If the estimate mean and variance over all possible values of differences between replicas delays and amplitude ratios $a_{2} / a_{1}$ are needed even the knowledge of probability distribution $p\left(\tau_{21} \mid a_{2} / a_{1}\right)$ is necessary. However, the amplitude of the closest replica is frequently comparable to that of the first one excluding the linear approximation shown above.

\section{Spectral estimator}

Our spectral estimator (a modified method of calculating the covariance matrix [16], its performance [17]) is nonlinear, however in the literature $(e g[7])$ it was derived a simple relation for the $\mathrm{CRB}$ in the case of using this method to estimate the spectrum of signals by $M$ samples at a high SNR. Although the exact relationship depends on the selected model order $P$ (order of the covariance matrix of the signal), the general formula has a broad minimum around $P=M / 3$

$$
\operatorname{var}_{\mathrm{SAM}}(\hat{\tau})=\frac{9}{8} \mathrm{CRB}_{M}(\hat{\tau})
$$

As can be seen, this value is very close to the minimum possible variance given by Cramer - Rao bounds (CRB).

\section{Multipath channel model for indoor propagation}

Errors due to multipath could be largely removed if you know the exact layout of the building. In practice, however, it is dubious whether the models of buildings will be available in those critical situations where the use of the proposed system is required. Instead for our estimates of the multipath impact we use a general statistics of the signal propagation in buildings. From there we determine the mean error of the direct path signal time of arrival and its variance.

Statistics of signal propagation in buildings are addressed in many sources. In [8] eg they deal with statistical modeling of UWB signal propagation in office buildings. From the measurements of pulses with a resolution of 2 ns they create a stochastic model of the signal power distribution around the building. The authors pay attention mainly to the total received power at different points of the building. Also other authors [9], [10] are dealing with the received signal power dependence on the distance or the delay time. In [11] the received signal distribution response on the building occupancy by humans is studied.

We have developed a simple model of the closest replica amplitude distribution in buildings. The environment studied in this paper usually contains a number of horizontal and vertical signal reflecting obstacles (walls, floors, roofs). The channel with the continuously distributed multipath is generally described by the following formula

$$
s_{2}(t)=\int_{0}^{\infty} \alpha(\tau, t) s_{1}(t-\tau) \mathrm{d} \tau
$$

where $s_{1}(t)$ and $s_{2}(t)$ input and output signal, $\alpha(\tau, t)$ is a channel impulse response, dependent on the replica delay $\tau$ and (generally) on time $t$.

In our case the time dependence of the channel response caused by the Doppler effect can be neglected but the channel response $\alpha(\tau)$ is a random zero mean function of the transmitter and the receiver positions $P_{T}$ and $P_{R}$. The channel response profile $P_{\alpha}(\tau)$ ie the replica power dependence on the replica delay in buildings have been measured and modeled in a number of publications (eg [13], [14]). Theoretical models of this profile, based on the ray model are usually exponential ([13]).

Presented models with continuous distribution of replicas successfully used for communication are suited for propagation over a large distance and for the narrow bandwidth systems (with low time-resolution), ie for systems with a high ratio between the total time of propagation and the time resolution. In our case we are interested in the influence of the closest replica for accurate determination of time of arrival of the direct signal at small distances. Therefore, we conducted our own (non-linear) derivation of immediate impact of the nearest replicas on the time of arrival measurement error based on the first replica delay probability. From there, we can calculate mean of the time of arrival error estimation and its variance due to the multipath propagation.

We will estimate the probability density $p(\tau)$, that the closest replica will be delayed behind the direct signal by $\tau$. We are starting with a ray model of two nearby paths: the direct signal path and the path of the signal reflected from the nearest plane obstacle. The situation is depicted in Fig. 1. The point A represents the transmitter 


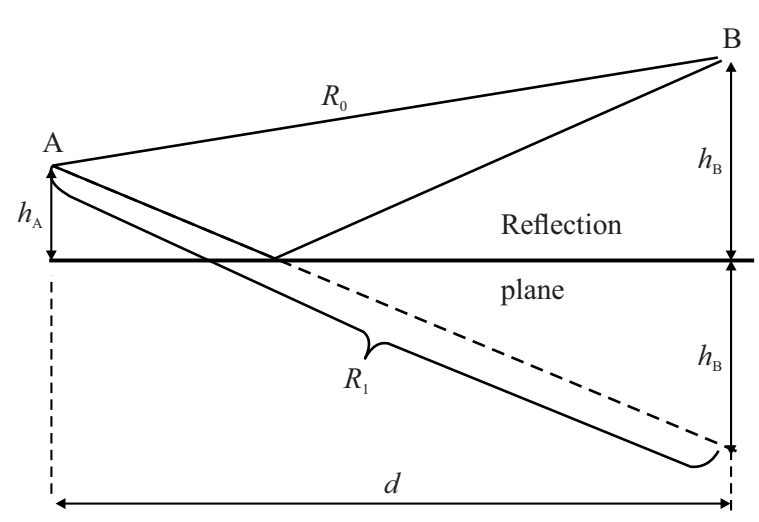

Fig. 1. Scheme for calculating the difference between paths of direct and reflected beam

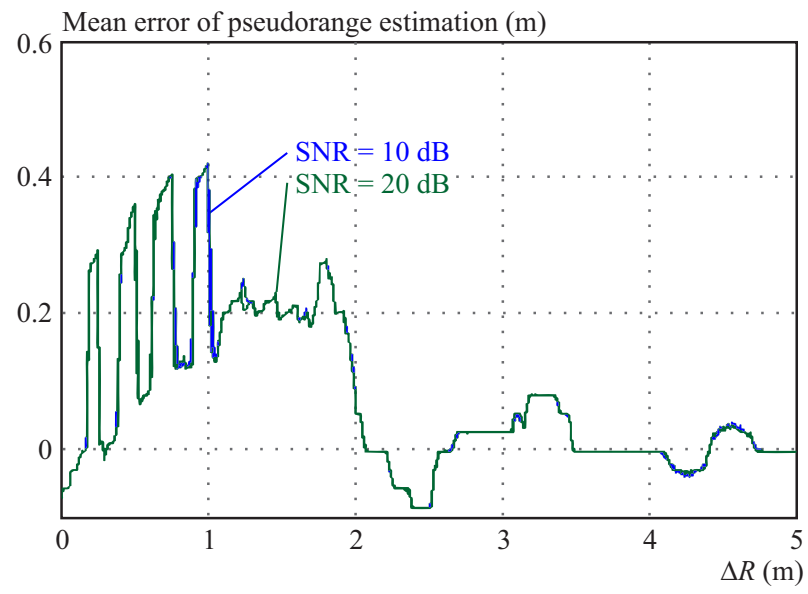

Fig. 3. Position estimation error due to the nearest replica separated by $\Delta R$ calculated by the CCF method averaged through replica relative phases in the range $0-2 \pi$

position within the building and the point $\mathrm{B}$ the receiver position in the area outside the building. For The distance difference $\Delta R$ between the direct and reflected signals can be expressed as follows

$\Delta R \equiv R_{1}-R_{0}=\sqrt{d^{2}+\left(h_{B}+h_{A}\right)^{2}}-\sqrt{d^{2}+\left(h_{B}-h_{A}\right)^{2}}$

where the meaning of $R_{1}, R_{0}, d, h_{A}$ a $h_{B}$ is obvious from Fig. 1.

Time difference of arrival of the two beams at the receiver will then be equal to: $\tau_{12}=\Delta R / c$, with $c$ being the light velocity. The probability $P(\Delta R)$ will be proportional to the ratio of the number of possible combinations of receiver and transmitter positions $N(\Delta R)$ differing by $\Delta R$ to $N_{\text {all }}$ the total number of possible combinations of positions of receiver and transmitter. Because we are concerned in the replica, closest to the direct signal, we only need to consider reflections from the wall (floor, ceiling) closest to the transmitter A. Therefore, the distance $h_{A}$ changes only in the range from 0 to the half of the

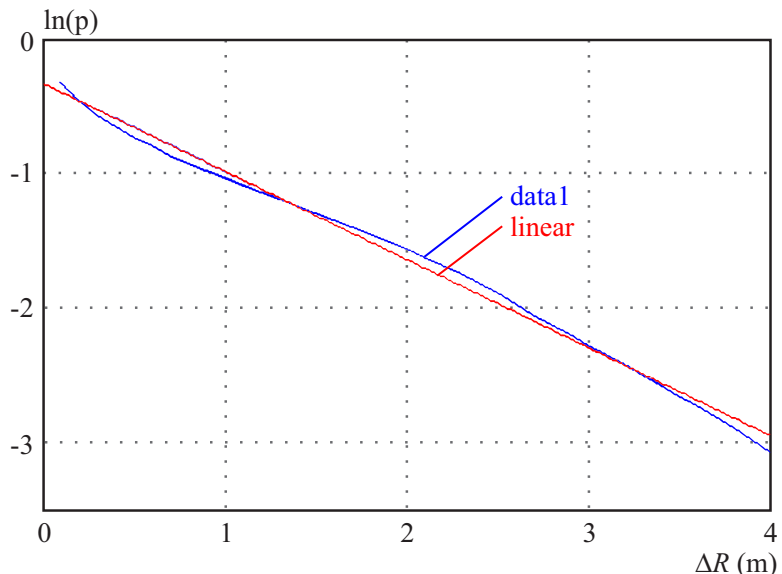

Fig. 2. PDF of the first replica compared to the exponential response in logarithmic scale

distance between two parallel walls in a building. Probability density $p\left(\tau_{12}\right)$ is obtained normalizing the variable $P(\Delta R)$

$$
p(\Delta R)=\frac{P(\Delta R)}{\int_{0}^{\Delta R_{\max }} P(\Delta R) \mathrm{d} \Delta R}
$$

This calculation was performed numerically for $h_{A} \in<$ $0,2.5 \mathrm{~m}>$. The calculated probability distribution response $p(\Delta R)$ on the closest replica delay $\Delta R$ in a logarithmic scale is shown in Fig. 2. It is apparent that also here we get approximately an exponential curve

$$
p(\Delta R) \cong \alpha \exp (-\alpha \Delta R)
$$

The numerically derived dependence is used for further calculation of the mean and variance of errors due to multipath propagation.

\subsection{Correlation estimator}

The correlation estimator was tested by simulation on a model that assumes the presence of only the direct signal and one (the nearest) replica of the same amplitude but with a variable phase. In simulations the time separation and phase difference of the direct signal and the replica were changed and the induced error in the time of arrival estimation of the direct signal was evaluated. These simulations were carried out for two values of signal-to-noise ratio and for 11 values of a relative replica phase in the range from 0 to $\pm \pi$.

Figure 3 shows the results for the correlation estimator (method $\mathrm{CCF}$ ). In the diagram the error mean value averaged through relative replica phases for the specific replica separation $\mathrm{d} R$ is plotted. We can see almost no dependence on the signal to noise ratio, since the CCF method is characterized by a high level of noise integration. At first the error increases linearly with the replica distance $\mathrm{d} R$ till approximately 2 meters, then the method resolves the two signals and the error abruptly falls down. 


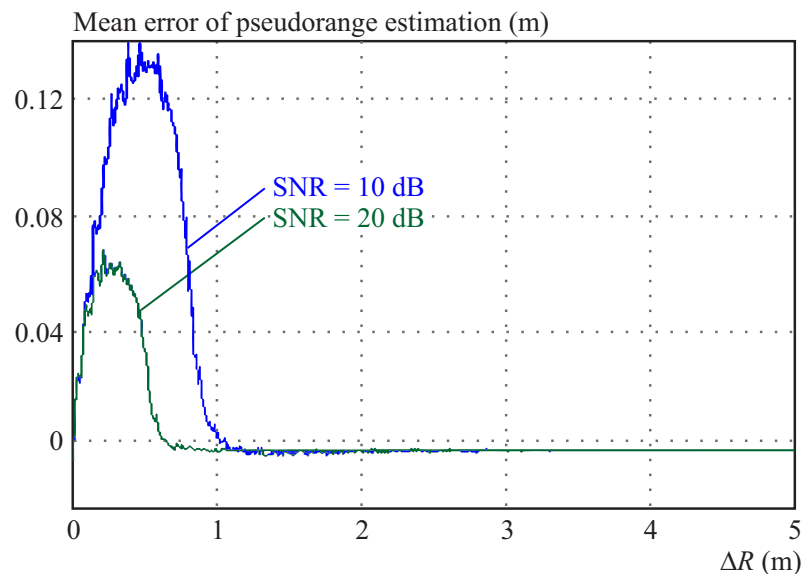

Fig. 4. Position estimation error due to the nearest replica separated by $\mathrm{d} R$ calculated by the SAM method

Using a probability distribution of the replica delay expressed by the beam paths difference $\Delta R$ - the total mean error (averaged through the replica distance and its relative phase) and the variance of the time of arrival measurement can be calculated

$$
\begin{aligned}
\mu_{\Delta R} & =\int_{0}^{\Delta R_{\max }} p(\Delta R) \varepsilon(\Delta R) \mathrm{d} \Delta R \\
\sigma_{\Delta R} & =\int_{0}^{\Delta R_{\max }} p(\Delta R)\left[\varepsilon(\Delta R)-\mu_{\Delta R}\right]^{2} \mathrm{~d} \Delta R
\end{aligned}
$$

Results of these calculations are in Tab. 1.

\subsection{Spectral estimator}

Figure 4 shows the behavior of errors calculated by the spectrum analysis method (SAM) - a method based on pseudospectrum analysis - in this case particularly the modified covarinace matrix method was used, $[16,17]$ - at various signal-to-noise ratios. Again the error mean values averaged through relative replica phases for the specific replica separation $\Delta R$ are plotted. Once again the error tends to increase linearly up to the value at which the method resolve the replica and the direct signal and then falls to zero. In this case the signal-to-noise ratio affects not only the error magnitude, but also the method resolution.

Table 1. The values of total mean errors and standard deviations due to the multipath propagation

\begin{tabular}{lcccc}
\hline $\begin{array}{l}\text { S/N } \\
(\mathrm{dB})\end{array}$ & $\begin{array}{c}\text { Mean } \\
\text { error } \\
(\mathrm{m}) \text { CCF }\end{array}$ & $\begin{array}{c}\text { Mean } \\
\text { error } \\
(\mathrm{m}) \text { SAM }\end{array}$ & $\begin{array}{c}\text { Standard } \\
\text { deviation } \\
(\mathrm{m}) \mathrm{CCF}\end{array}$ & $\begin{array}{c}\text { Standard } \\
\text { deviation } \\
(\mathrm{m}) \text { SAM }\end{array}$ \\
\hline 10 & 0.136 & 0.042 & 0.442 & 0.064 \\
20 & 0.136 & 0.015 & 0.443 & 0.030 \\
\hline
\end{tabular}

The results of the calculations summarized in Table. 1 shows that the mean errors of the direct signal delay estimation due to the multipath propagation is non-zero and positive for the both methods. We can see also, that the SAM method shows much better mean errors and variances then the CCF method.

\section{Conclusion}

In the article an analysis of the signal time arrival estimation errors due to multipath propagation in complicated environments is presented. Two methods were tested for the delay estimation $i e$ : the correlation method utilizing cross the correlation of the received signal and the model of the transmitted signal and the spectral analysis method, using a modified method of covariance matrix calculation.

To estimate the probability $P(\tau)$, that the closest replica will be delayed by behind the direct signal a simple channel model with two-path propagation inside building was created. From there the mean error and variance of the time of arrival estimation caused by multipath propagation was calculated. Finally we have found, that the SAM method shows much better mean errors and variances then the $\mathrm{CCF}$ method.

\section{Acknowledgements}

The research, described in the paper was supported by the Czech Ministry of Industry and Trade, project No. FV10486.

\section{REFERENCES}

[1] L. Zaplatilek, M. Pola and P. Bezousek, "Modeling of precise indoor positioning system", Conference on Microwave Techniques (COMITE) [online], IEEE, 2015, 1-4 [cit.2015-06-15].DOI: 10. 1109/ COMITE.2015.7120322. ISBN 978-1-4799-8121-2.

[2] S. H. Gezici and V. Poor, "Position Estimation via Ultra-WideBand Signals", Proceedings of the IEEE [online], 2009, 97 (2), 386-403 [cit.2016-07-04], DOI: 10.1109/JPROC.2008. 2008840. ISSN 0018-9219.

[3] A. N. d'Andrea, U. Mengali and R. Reggiannini, "The modified Cramer-Rao bound and its application to synchronization problems", IEEE Transactions on Communications [online]. 1994, 42(2/3/4), 1391-1399 [cit.2016-04-26], DOI: 10.1109/TCOMM, 1994, 580247, ISSN 0090-6778.

[4] J.-P. [4] and Delmas, "Closed-form expressions of the true Cramer-Rao bound for parameter estimation of BPSK, MSK or QPSK waveforms", 14th European Signal Processing Conference (EUSIPCO 2006), Florence, Italy, EURASIP, 2006, pp. 4-8, ISSN 2219-5491.

[5] D. [5], Ch-Ch. Dardari, M. Chong and Win, "Improved Lower Bounds on Time-of-Arrival Estimation Error Realistic UWB Channels", 2006 IEEE International Conference on Ultra- Wideband [online]. IEEE, 2006, pp. 531-537 [cit.2016-07-05].DOI: 10.1109/ICU, 2006, 281605, ISBN 1-4244-0102-X.

[6] J. [6], L. Serra, M. Blanco and Najar, "Cramer-Rao bound for time-delay estimation the frequency domain", 17th European Signal Processing Conference [online], 2009, pp. 1037-1041 [cit.2016-07-05], ISSN 2219-5491.

[7] S. [7], J. Lang, McClellan, Frequency, estimation, with, maximum, entropy and spectral, ", IEEE Transactions on Acoustics, 
Speech, and Signal Processing [online], 1980, 28(6), pp. 716-724 [cit.2016-07-05], DOI: 10.1109/TASSP, 1980, 1163467, ISSN 0096-3518.

[8] D. Cassioli, M. Z. Win and A. F. Molisch, "The ultra-wide bandwidth indoor channel: from statistical model to simulations", IEEE Journal on Selected Areas Communications [online]. 2002, 20(6), pp. 1247-1257 [cit.2016-05-10].DOI: 10.1109/JSAC.2002 .801228.ISSN 0733-8716.

[9] A. Moscchitta, D. Macii, F.Trenti, S. Dalpez and A. Bozzoli, "Characterization of a geometrical wireless signal propagation model for indoor ranging techniques", In: 2012 IEEE International Instrumentation and Measurement Technology Conference Proceedings [online], IEEE, 2012, pp. 2598-2603, [cit.2016-05-10], DOI: 10.1109/I2MTC，2012，6229373，ISBN 978-1-4577-1772-7.

[10] R. Desimone, B. M. Brito and J. Baston, "Model of indoor signal propagation using log-normal shadowing", In: 2015 Long Island Systems, Applications and Technology [online], IEEE, 2015, pp. 1-4 [cit.2016-05-10], DOI: 10.1109/LISAT, 2015, 7160217, ISBN 978-1-4799-8643-9.

[11] Y.-H. Kim, J.-H. Lee and S.-Ch. Kim, "Modelling of UWB channel with population density indoor LOS environments", IEEE Antennas and Wireless Propagation Letters [online], pp. 1-1 [cit.2016-05-10], DOI: 10.1109/LAWP, 2015, 2512988, ISSN $1536-1225$.

[12] J. Sykora, Theory of digital communication (in Czech)", Praha, CVUT Publ., 2002, ISBN 80-01-02478-4.

[13] J. Hansen, "An analytical calculation of power delay profile and delay spread with experimental verification", IEEE Communications Letters [online], 2003, 7(6), pp. 257-259 [cit.2016-06-23], DOI: 10.1109/LCOMM, 2003, 813815, ISSN 1089-7798.

[14] S. S. Ghassemyadeh, L. J. Geenstein, T. Sveinsson, A. Kavcic and V. Tarokh, "UWB Delay Profile Models for Residential and Commercial Indoor Environments", IEEE Transactions on Vehicular Technology[online], 2005, 54(4), pp. 1235-1244, [cit.2016-06-23], DOI: 10.1109/TVT, 2005, 851379, ISSN 00189545.
[15] E. D. Kaplan and C. Hegarty, "Understanding GPS: principles and applications", 2nd ed. , Boston: Artech House, c2006, Prentice Hall signal processing series, ISBN 15-805-3894-0.

[16] A. Vizinho and L. R. Wyatt, "Evaluation of the use of the modified-covariance method HF radar ocean measurement", IEEE Journal of Oceanic Engineering [online], 26(4), pp. 832-840 [cit.2016-07-05], DOI: 10.1109/48.972123, ISSN 03649059.

[17] M. Lauterbach, M. Pola, L. Zaplatilek and P. Bezousek, "Accuracy of spectral analysis methods for precision indoor positioning system", In: The 10th International Conference on Digital Technologies 2014 [online], IEEE, 2014, pp. 217-220 [cit.2015-06-12].DOI: 10.1109/DT.2014.6868716, ISBN 978-14799-3303-7.

18] J. Skapa and M. Pola, "Ultra Wide Band receiver design", 2016, In: 26th International Conference Radioelektronika, Kosice, Slovakia, 2016, pp. 304-308, DOI: 10.1109/RADIOELEK.2016. 7477406 .

Received 16 December 2016

Marek Pola was born in Třebíč, Czechoslovakia in 1984. He received his BS degree from the Czech Technical University in Prague in 2007, his MS degree from the University of Pardubice in 2009 and his $\mathrm{PhD}$ degree from the same university in 2016. Since 2010 he is employed as an assistant professor at the Faculty of Electrical Engineering and Informatics of the University of Pardubice where he is engaged with the multilateration systems design and radar signal processing.

Pavel Bezoušek was born in Ostrava, Czechoslovakia in 1943. He received his MS degree from the Czech Technical University in Prague in 1966 and his $\mathrm{PhD}$ degree from the same university in 1980. He was with the Radio Research Institute of the Tesla Pardubice from 1966 till 1994, where he was engaged in microwave circuits and systems design. Since then he is with the University of Pardubice, now at the Faculty of Electrical Engineering and Informatics. Presently he is engaged in radar systems design. 